\title{
The Effect of Using a Food and Fitness iPad Application to Increase Obesity Awareness in Pre-licensure Nursing Students
}

Karen Aul

Department of Nursing, Arkansas State University, P. O. Box 910, State University, AR 72467, USA

\begin{abstract}
Background: Obesity is a major health issue that increases medical costs and decreases the quality of life. It is therefore important to try to prevent obesity by adhering to a healthy lifestyle. The definition of a healthy lifestyle may be very different for individuals in their perception of obesity. The purpose of this study was to examine the perceptions of healthy eating and activity patterns of pre-licensure nursing students in order to increase their obesity awareness.

Methods: A pre-test questionnaire on healthy eating and activity patterns was administered to a convenience sample of 44 pre-licensure nursing students. The students then tracked their eating and activity patterns for one week using the iPad application of ChooseMyPlate Tracker by Live strong.com and a post-test was given to those nursing students who used the application. Only half of the students who tracked their eating and activity patterns had received nutrition training $(n=22)$. The data was analyzed for variances using descriptive statistics and the Mann-Whitney $U$ test.

Results: There was a significant difference $(\mathrm{p}<.05)$ between the pre-test and post-test scores. Nursing students had higher perceptions of healthy eating and activity after tracking their own eating and activity patterns for a week. There was no significant difference in the scores between the groups of nursing students who were provided nutrition training and those that did not receive the training.

Conclusion: Findings from this study indicate that nursing students had a greater awareness of a healthy lifestyle when they actively tracked their own eating and activity patterns. Nutrition training had no impact on how they perceived their healthy eating and activity patterns. These findings highlight how an iPad applicationcan promote healthy eating and activity in individuals to increase obesity awareness. This awareness has significant implications for the prevention of obesity.
\end{abstract}

\section{Introduction}

Obesity has been a rising epidemic over the past couple of decades with its prevalence increasing in both developed and developing nations. Globally, it is a major contributor to chronic disease and disability [1]. It remains one of the biggest threats to the health of the children and to America as a country. Obesity rates in America rose sharply from 1980 to 2000; adult rates doubled and children's rates tripled [2]. Change has been slow to decrease the rates of obesity, and obesity rates remain very high; in national surveys, more than $30 \%$ of adults and nearly $17 \%$ of children were found to be obese.

Reversing the obesity epidemic in America will require the encouragement of healthy eating and active living. Better policies are needed to change the course of the nation's obesity epidemic so that children will have the opportunity to grow into healthy adults. A culture of health needs to be built. Organizations need to advocate for healthier lifestyles, such as good nutrition and daily exercise, to curb this growing epidemic. One type of organization that can be instrumental in preventing obesity is the academia environment. College students who are entering a health profession can receive training on healthier lifestyles, and in turn educate their patients.

Robiner, Lazear, and Duffy [3] recommend the development of programs to train health profession students on weight management through healthy lifestyle interventions. Research by Williamson and Kautz [4] suggested a way to increase awareness of the health risks of obesity among African-American nursing students through an hourlong educational program. According to Levy et al. [5], simulation models may be a method to understand how the behaviors of diet and activity affect health outcomes. These are all types of interventions that may be helpful. However, the perceptions of healthy lifestyle choices must also be considered. Research by Brown et al. [6] found influencing factors besides diet and physical activity that were associated with increases in body weight. However, it is difficult to separate the effects of the behaviors, health outcomes, and obesity rates. What is known is that healthy lifestyle choices is one of the most powerful tools to reduce the onset of disease; good nutrition is an important step in reducing chronic disease. Healthy behaviors promote the quality of life [7]. The aim of this study was to add to the awareness of obesity. By identifying the perception of obesity from a group of nursing students, the hope was for the students to be more cognizant of their own lifestyle choices. Through this recognition, they would be better able to educate their patients on the benefits of healthy eating and adequate activity.

\section{Materials \& Method}

This descriptive study used cross-sectional data from a survey questionnaire given as a pre-test and then as a post-test after the participants tracked their dietary and activity patterns for one week using a designated iPad application. The content items for the

"Corresponding Author: Dr. Karen Aul, Department of Nursing, Arkansas State University, P. O. Box 910, State University, AR 72467, USA, E-mail: kaul@astate.edu

Citation: Aul K (2017) The Effect of Using a Food and Fitness iPad Application to Increase Obesity Awareness in Pre-licensure Nursing Students. Int J Nurs Clin Pract 4: 224. doi: https://doi.org/10.15344/2394-4978/2017/224

Copyright: (C) 2017 Aul. This is an open-access article distributed under the terms of the Creative Commons Attribution License, which permits unrestricted use, distribution, and reproduction in any medium, provided the original author and source are credited. 
Citation: Aul K (2017) The Effect of Using a Food and Fitness iPad Application to Increase Obesity Awareness in Pre-licensure Nursing Students. Int J Nurs Clin Pract 4: 224. doi: https://doi.org/10.15344/2394-4978/2017/224

questionnaire were obtained from the ChooseMyPlate.gov recommendations for healthy eating from the United States Department of Agriculture [8]. ChooseMyPlate is a comprehensive, highly visual website that offers sample menus and recipes, daily food plans, super tracker tools and a personalized nutrition and physical activity plan [9]. Examples of the questionnaire items based on the specific ChooseMyPlate recommendations include: make half your plate fruits and vegetables, make at least half your grains whole, drink water instead of sugary drinks, switch to fat-free or low-fat milk, avoid oversized portions, and be active your way [10].

Study eligibility consisted of pre-licensure nursing students who had an iPad. A convenience sample of 62 sophomore nursing students in a baccalaureate nursing program at a university in the Midwestern United States was selected. All students were required to have iPads for their nursing courses. After receiving institutional review board approval, the researcher implemented the study.

The study purpose was explained, written informed consent given, and confidentiality and anonymity guaranteed. Of the 62 students, 18 declined to participate after learning of the time commitment to monitor their dietary intake and activity patterns for one week. Therefore, 44 participants represented the sample size. The participants were further grouped according to whether they received nutrition training in a previous nursing course. This training consisted of four hours of instruction regarding basic nutrition and clinical nutrition relevant to disease processes.

The researcher collected data using a pre-test questionnaire composed of 12 items that included questions on healthy eating and activity. The participants were asked to rate their perceptions of their eating and activity patterns on a Likert scale from 1 to 5 as follows; $1=$ almost never, $2=$ seldom, $3=$ about half the time, $4=$ often, and $5=$ almost always. After completing the pre-test questionnaire, the students were given instructions on downloading the free iPad application of ChooseMyPlate Tracker by Livestrong.com. This application was a user-friendly way for participants to track their dietary intake, daily calories, and level of physical activity. The participants had no questions and all agreed to track their eating and activity patterns using the iPad application for one week. One week later, the researcher met the participants and administered a posttest questionnaire. The post-test questionnaire was comprised of the same 12 items that were on the pretest, with the addition of 1 question regarding how well the participant tracked their nutrition and activity using the iPad application.

\section{Results and Discussion}

The quantitative data from the 44 surveys was analyzed using SPSS software. Descriptive statistics used two independent sample t-tests to analyze and compare the means for the two groups of nursing students; the group that received nutrition training $(n=22)$ and the group that did not receive nutrition training $(n=22)$. There was a significant difference $(\mathrm{p}<.05)$ between the means for the pre-tests and post-tests. The means for each item response was higher on the posttests as compared to the results from the pre-tests (Table 1).

The Mann-Whitney $\mathrm{U}$ test was used to determine any significance between the two groups of nursing students who were provided nutrition training and those that did not receive the training. The analysis showed no significant difference in the scores between the two groups. Bonferroni adjustment was further used to determine the level of significance as $\mathrm{p}<.004$.

The purpose of this research was to determine the perceptions of healthy eating and activity from a group of nursing students. In relation to other published work, Hassan et al. [11] found that the perception of obesity depended on the major of the college student; the science students had higher individual awareness and perceptions of obesity than the art students. In this study, although the participants were all nursing students who had science courses for their chosen major, nutrition training had no impact on how they perceived their healthy eating and activity patterns.

There is varying research findings on the factors that influence healthy eating and activity patterns in college students and young adults. A study by Harmon et al. [12] looked at perceptions of social influences that impacted college students' diet and physical activity. They found that high school friends were perceived as the least influential and significant others were perceived as the most influential on diet and physical activity behaviors. Jhaveri and Patki [13] examined behavioral factors affecting eating patterns, and found peer influence to be positively correlated with media exposure and negatively correlated with satisfaction. Research by Mathews et al. [1]

\begin{tabular}{|c|c|c|c|c|}
\hline Rating Scale & Pre-test & Pre-test & Post-test & Post-test \\
\hline $\begin{array}{l}1=\text { almost never } \\
2=\text { seldom } \\
3=\text { about half the time } \\
4=\text { often } \\
5=\text { almost always }\end{array}$ & $\begin{array}{l}\text { Mean } \\
\text { Nutrition } \\
\text { training } \\
\mathrm{n}=22\end{array}$ & $\begin{array}{c}\text { Mean } \\
\text { No } \\
\text { nutrition } \\
\text { training } \\
\mathrm{n}=22\end{array}$ & $\begin{array}{c}\text { Mean } \\
\text { Nutrition } \\
\text { training } \\
\mathrm{n}=22\end{array}$ & $\begin{array}{c}\text { Mean } \\
\text { No } \\
\text { nutrition } \\
\text { training } \\
\mathrm{n}=22\end{array}$ \\
\hline 1) Do you eat healthy? & 3.0000 & 3.2600 & 4.0000 & 4.1364 \\
\hline 2) Do you eat out at a restaurant or order take-out? & 3.1250 & 3.2581 & 3.5926 & 3.9091 \\
\hline 3) Do you read the Nutrition Facts Label on food products? & 2.8750 & 3.0645 & 3.7407 & 4.0909 \\
\hline 4) Do you eat oversized portions? & 3.3125 & 2.7333 & 3.4815 & 3.8636 \\
\hline 5) Do you make half your plate fruits and vegetables? & 2.4063 & 2.6452 & 3.3704 & 3.5909 \\
\hline 6) Do you choose whole-grain food products when available? & 3.4375 & 3.3871 & 3.3704 & 4.0952 \\
\hline 7) Do you eat sugary and high fat desserts? & 3.1875 & 2.7097 & 3.7037 & 4.0455 \\
\hline 8) Do you choose fat-free or low fat milk and dairy products? & 2.8438 & 3.1290 & 3.2222 & 3.8571 \\
\hline 9) Do you choose lean protein such as lean beef, pork, chicken, turkey, beans, or tofu? & 3.8438 & 4.0000 & 4.0000 & 4.3182 \\
\hline 10) Do you drink water instead of sugary drinks? & 3.7500 & 4.0000 & 4.4444 & 4.5909 \\
\hline 11) Do you stay physically active for at least 2.5 hours a week? & 3.5625 & 3.5484 & 4.1852 & 4.4545 \\
\hline 12) Do you feel that you are active enough to maintain good health? & 3.1875 & 3.4516 & 4.2593 & 4.5455 \\
\hline 13) How well did you track your nutrition and exercise using the iPad application? & & & 3.3333 & 3.7273 \\
\hline
\end{tabular}


Citation: Aul K (2017) The Effect of Using a Food and Fitness iPad Application to Increase Obesity Awareness in Pre-licensure Nursing Students. Int J Nurs Clin Pract 4: 224. doi: https://doi.org/10.15344/2394-4978/2017/224

Page 3 of 3

found no significant relationship between the knowledge and attitude of college students regarding obesity. However, the students' knowledge and positive attitudes were enhanced with the implementation of an obesity awareness program. It was recommended that awareness programs are needed to provide information on healthy food habits, healthy lifestyles, and a physically active daily routine. This work is significant as it contributes research to the importance of increased obesity awareness in students. Previous studies has demonstrated the need for obesity awareness. In this study, nursing students had a greater awareness of a healthy lifestyle when they actively tracked their own eating and activity patterns. An awareness of healthy eating and activity is necessary for nursing students to be effective educators and advocates for their patients.

\section{Conclusions}

In conclusion, this study suggests how an iPad application can promote healthy eating and activity in nursing students to increase obesity awareness. It is hoped that findings from this research emphasize the need to create more opportunities for health professionals to acquirean obesity awareness. Developing awareness programs in health profession education programs can have significant implications for the future prevention of obesity. Awareness programs can address lifestyle changes in the lives of health professional students as well as in their practice. The education and training that the health professional students receive can be used to educate patients on integrating healthy lifestyle interventions. Achieving healthier lifestyles may lead to better health outcomes.

\section{Competing Interests}

The author declares that the she has no competing interests.

\section{References}

1. Mathews A, Mathias J, Thomas UM, Williams S, Noronha AV (2015) An exploratory and evaluative study of the prevalence, related knowledge, attitude, and effectiveness awareness program regarding obesity among students of selected degree colleges in Mysore City. Asian Journal of Nursing Education and Research 5: 167-171.

2. Trust for America's Health and the Robert Wood Johnson Foundation (2015) The State of Obesity: Better Policies for a Healthier America.

3. Robiner W, Lazear S, Duffy B (2013) Promoting health behaviours in medical education. The Clinical Teacher 10: 160-164.

4. Williamson W, Kautz DD (2013) Increasing awareness in African-American BSN students of the health risks of obesity. The ABNF Journal 24: 40-41.

5. Levy DT, Mabry PL, Wang YC, Gortmaker S, T-KHuang T, et al. (2011) Simulation models for obesity: A review of the literature and implications for research and policy. Obesity Rev 12: 378-394.

6. Brown RE, Sharma AM, Ardern CI, Mirdamadi P, Kuk JL (2015) Secular differences in the assoiciation between caloric intake, macronutrient intake, and physical activity with obesity. Obes Res Clin Pract 10: 243-255

7. Healthy People 2020 (2017) The Mission, Vision, and Goals of Healthy People 2020.

8. United States Department of Agriculture (USDA) (2016) ChooseMyPlate.

9. Palumbo C (2013) Best of MyPlate tracking tools and apps. Journal of the American Academy of Physician Assistants 26: 5-5

10. Robert Wood Johnson Foundation (2015) Information to make choices Dietary guidelines for Americans, food and menu labeling, and marketing standards.

11. Hassan S, Rahman NAA, Ghazali K, Ismail N, Budin K (2014) Perception on obesity among university students: A case study using factor analysis. AIP Conference Proceedings 1605: 973-978.
12. Harmon BE, Forthofer M, Bantum EO, Nigg CR (2016) Perceived influence and college students' diet and physical activity behaviors: An examination of ego-centric social networks. BioMed Central Public Health 16: 473.

13. Jhaveri SS, Patki SM (2016) Locus of control, peer influence on dieting, media exposure and body image satisfaction in young adults. Indian Journal of Health and Wellbeing 7: 369-372. 